\title{
Creative method of removing a giant fibrovascular polyp under endoscopic guidance
}

A 59-year-old man was admitted to the Beijing Friendship Hospital with progressive dysphagia for solid foods. He had been coughing for 20 days, which resulted in the repeat regurgitation of the polyp into his mouth followed by swallowing of the polyp [1]. Results of laboratory tests on admission were negative.

Endoscopy revealed a large polyp stalk protruding into the lumen from Killian's triangle. The pedunculated polyp was smooth and covered with normal mucosa, with some congestion at its top segment. The polyp was about $15 \mathrm{~cm}$ in length. Endoscopic ultrasonography showed the polyp with medium signal intensity. The base was located at the entrance to the cervical esophagus, and the polyp extended to the lower thoracic segment of the esophagus. These findings suggested a diagnosis of fibrovascular polyp.
The patient underwent endoscopic polypectomy under general anesthesia with elective intubation and airway protection. An attempt to insert a nylon loop to snare the projection was unsuccessful because the projection was too thick ( $\bullet$ Fig. 1 a). We therefore snared part of the giant polyp, and dragged it out into the oral cavity ( $\bullet$ Fig. 1 b), while simultaneously withdrawing the endoscope. The polyp was fixed with a towel clamp ( $\bullet$ Fig. 1 c), and the endoscope was inserted into the mouth to observe the polypectomy ( $\bullet$ Fig. 1d). Finally, the base of the polyp was snared using a nylon loop under endoscopic guidance ( $\bullet$ Fig. 1 e), and the giant polyp was resected ( $\bullet$ Fig. 1 f, - Video 1). The wound was closed with hemostatic forceps. Minor bleeding occurred but ceased rapidly.

In conclusion, this case demonstrates that giant fibrovascular polyps can be resected under endoscopic guidance. The polypectomy method described can be used to resect thick and long polyps. This procedure may make it easier to resect giant polyps and is technically less demanding than procedures previously reported by others [2-4].

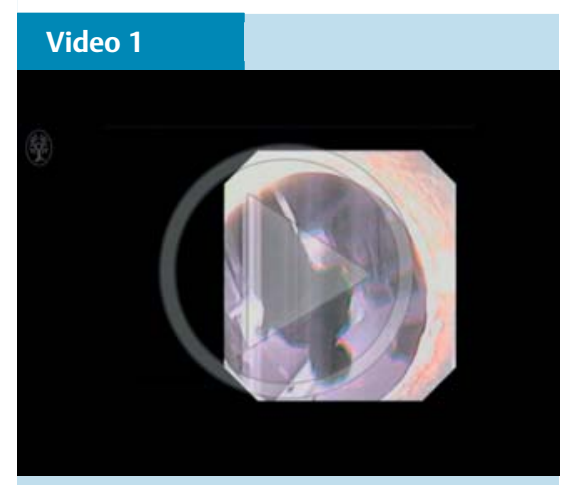

A novel approach to the removal of a giant pedunculated polyp under endoscopic guidance. It was not feasible to deploy the endoloop from inside the esophagus. A useful method was applied whereby the polyp was dragged into the oral cavity, and the endoscope was used to view and guide the polypectomy from the mouth. Finally, the giant polyp was resected using a nylon loop.
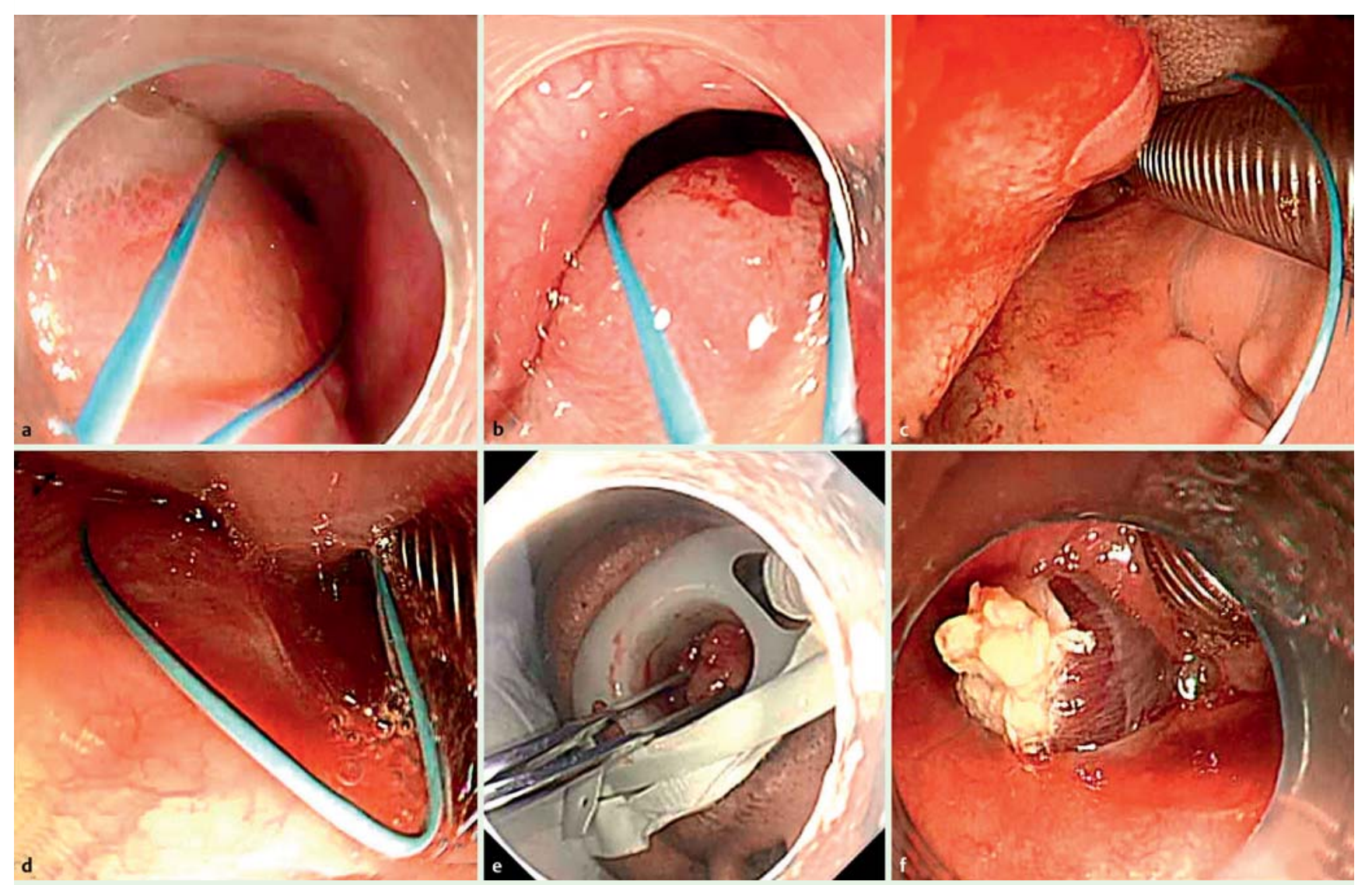

Fig. 1 Removal of a giant fibrovascular polyp under endoscopic guidance. a The stalk of the polyp was too thick to snare with a nylon loop. b The nylon loop was used to snare part of the polyp. c A towel clamp was used to grasp the giant polyp and to fix it in place. $\mathbf{d}$ The endoscope was used to view and guide the polypectomy in the oral cavity. e The nylon loop was used to snare the base of the projection. $\mathbf{f}$ The residual polyp in the esophagus after polypectomy. 
Endoscopy_UCTN_Code_TTT_1AO_2AG

Competing interests: None

\section{Wei Jiang*, Yongjun Wang*, Peng Li, Fujing Lv, Wei Li, Ming Ji, Shutian Zhang}

Department of Gastroenterology, Beijing Friendship Hospital, Capital Medical University, Beijing, China

* These authors contributed equally to this work.

\section{References}

1 Caceres M, Steeb G, Wilks SM et al. Large pedunculated polyps originating in the esophagus and hypopharynx. Ann Thorac Surg 2006; 81: 393-396

2 Jung EY, Choi SO, Cho KB et al. Successful endoscopic submucosal dissection of a giant polyp in a 21-month-old female. World J Gastroenterol 2014; 20: 323-325

3 Gupta M, Chaudhary N, Gupta M. Fibrovascular polyp of the oropharynx. Singapore Med J 2011; 52: e35-36

4 Jose P, Scott N, Sarela AI. Two-stage removal of giant fibrovascular polyp of the oesophagus. BMJ Case Rep 2010. doi: 10.1136/ bcr.05.2010.3011
Bibliography

Dol http://dx.doi.org/

$10.1055 / \mathrm{s}-0042-111318$

Endoscopy 2016; 48: E242-E243

(c) Georg Thieme Verlag KG

Stuttgart · New York

ISSN 0013-726X

Corresponding author Shutian-Zhang, MD

Department of Gastroenterology Beijing Friendship Hospital

Capital Medical University

95 Yongan Road

Beijing

China

Fax: $+86-10-63138338$

zhangshutian@ccmu.edu.cn 